\title{
Antianemic Treatment of Cancer Patients in German Routine Practice: Data from a Prospective Cohort Study_-The Tumor Anemia Registry
}

\author{
Tilman Steinmetz, ${ }^{1}$ Jan Schröder, ${ }^{2}$ Margarete Plath, ${ }^{3}$ Hartmut Link, ${ }^{4}$ \\ Michèle Vogt, ${ }^{5}$ Melanie Frank, ${ }^{5}$ and Norbert Marschner ${ }^{6}$ \\ ${ }^{1}$ Outpatient Clinic for Hematology and Oncology, Sachsenring 69, 50677 Cologne, Germany \\ ${ }^{2}$ Outpatient Clinic for Oncology, Kettwiger Strasse 62, 45468 Mülheim an der Ruhr, Germany \\ ${ }^{3}$ Outpatient Clinic for Oncology, Prinzregentenstrasse 1, 86150 Augsburg, Germany \\ ${ }^{4}$ Department for Internal Medicine I, Westpfalz-Klinikum, Hellmut-Hartert-Strasse 1, 67655 Kaiserslautern, Germany \\ ${ }_{5}^{5}$ OMEDICO, Hanferstrasse 28, 79108 Freiburg, Germany \\ ${ }^{6}$ Outpatient Clinic for Interdisciplinary Oncology and Hematology, Wirthstrasse 11c, 79110 Freiburg, Germany
}

Correspondence should be addressed to Tilman Steinmetz; steinmetz@oncokoeln.de

Received 1 October 2015; Revised 3 December 2015; Accepted 14 December 2015

Academic Editor: Bruno Annibale

Copyright (C) 2016 Tilman Steinmetz et al. This is an open access article distributed under the Creative Commons Attribution License, which permits unrestricted use, distribution, and reproduction in any medium, provided the original work is properly cited.

\begin{abstract}
The aim of this prospective cohort study was to assess current antianemic treatment of cancer patients in German routine practice, including diagnostics, treatments, and quality of life (QoL). 88 study sites recruited 1018 patients at the start of antianemic treatment with hemoglobin $(\mathrm{Hb})$ levels $<11 \mathrm{~g} / \mathrm{dL}$ (females) or $<12 \mathrm{~g} / \mathrm{dL}$ (males). Patients were followed up for 12 weeks. $63 \%$ of the patients had inoperable solid tumors, $22 \%$ operable solid tumors, and 15\% hematological malignancies. Over $85 \%$ received chemotherapy. Median age was 67 years; $48 \%$ were male. Red blood cell transfusions (RBCTx) were given to 59\% of all patients and to $55 \%$ of the patients with $\mathrm{Hb} \geq 8 \mathrm{~g} / \mathrm{dL}$ on day 1 of the observation period (day 1 treatment). Erythropoiesis-stimulating agents (ESAs) were the second most frequently applied day 1 treatment (20\%), followed by intravenous (IV) iron (15\%) and ESA + IV iron (6\%). Only about a third of patients were tested for blood serum iron parameters at the start of treatment. Overall, more than half of the patients had long-term responses to antianemic therapy. Our data suggest that in routine practice diagnostics for treatable causes of anemia are underused. A high proportion of cancer patients receive RBCTx. It should be discussed whether thorough diagnostics and earlier intervention could decrease the need for RBCTx. This trial is registered with NCT01795690.
\end{abstract}

\section{Introduction}

Anemia is defined as a hemoglobin $(\mathrm{Hb})$ level of $<12 \mathrm{~g} / \mathrm{dL}$ for nonpregnant women and $<13 \mathrm{~g} / \mathrm{dL}$ for men, according to the World Health Organization [1]. It is a common complication of multifactorial etiology among patients with malignant diseases. The European Cancer Anemia Survey (ECAS) reported an overall anemia incidence $(\mathrm{Hb}<12 \mathrm{~g} / \mathrm{dL})$ of more than $50 \%$ during the 6-month survey period for patients with solid or hematological tumors who received their first anticancer treatment. Anemia incidence was almost $65 \%$ in patients receiving chemotherapy [2]. Low Hb levels are associated with poor physical performance status [2-5] and decreased quality of life (QoL) [3, 6-9], indicating a need for early antianemic treatment.

Treatment strategies include red blood cell transfusions (RBCTx), erythropoiesis-stimulating agents (ESAs), and iron supplementation either alone or in combination with ESAs. Treatment decision-making should be based on the best benefit-to-risk ratio for each patient and depends on patients' $\mathrm{Hb}$ level, the presence of symptoms, and the underlying cause for anemia as evaluated by blood parameters such as ferritin, transferrin saturation (TSAT), folate, and vitamin B12 [1012]. While the National Comprehensive Cancer Network 
(NCCN) has published a comprehensive guideline on anemia management, current guidelines in Europe focus on the application of ESAs and/or transfusions. The administration of intravenous (IV) iron is the treatment of choice for cancer patients with anemia due to absolute iron deficiency (AID). It has been shown to improve efficacy and is thus recommended in combination with ESAs for patients with functional iron deficiency (FID) [10-12]. If iron deficiency is excluded, the European Organization for Research and Treatment of Cancer (EORTC) recommends ESAs to treat symptomatic anemia with Hb levels $\geq 9 \mathrm{~g} / \mathrm{dL}$ and to assess whether transfusions are required in case of $\mathrm{Hb}$ levels $<9 \mathrm{~g} / \mathrm{dL}$ [10]. The NCCN advises thorough diagnostics for possible causes of anemia and subsequent treatment of these. If no treatable cause can be identified, transfusions are recommended depending on the presence of symptoms and comorbidities. ESAs are suggested for anemic patients undergoing palliative cancer treatment but not for patients receiving chemotherapy with curative intent [12]. The German guideline on the use of transfusions considers them an option depending on severity and symptoms of anemia, especially when rapid, short-term improvement of $\mathrm{Hb}$ levels $<8 \mathrm{~g} / \mathrm{dL}$ is required [13].

Prospective, observational studies can be used to assess the current state of care. In 2001/2002, the ECAS assessed prevalence, incidence, and treatment of anemia in more than 15,000 cancer patients in Europe. Over all patients, ESA therapy was the most frequently used antianemic treatment, while transfusions were most commonly applied in anemic patients with $\mathrm{Hb}$ levels $\leq 9.9 \mathrm{~g} / \mathrm{dL}$ receiving chemotherapy [2]. In 2004/2005, the German Cancer Anemia Registry (CAR) was a survey on the planned anemia management of almost 2,000 cancer patients in German routine care. Overall, the three predefined treatment strategies "to correct underlying disorder causative of anemia" (e.g., iron or vitamin deficiency or bone marrow infiltration), "to use transfusions as firstline treatment," and "to use ESA as first-line treatment" were selected equally frequently, while diagnostic measures were used in two-thirds of patients only [3].

Here, we present data on the current anemia management in cancer patients from the Clinical Tumor Anemia Registry (TAR) conducted in 2012/2013. This paper addresses the treatment reality of patients with cancer and/or therapy related anemia, the use of diagnostic measures, and effectiveness of treatment based on changes in $\mathrm{Hb}$ values and $\mathrm{QoL}$ within three months after the start of antianemic treatment.

\section{Patients and Methods}

2.1. Study Design. The TAR was an open, prospective, multicenter, longitudinal, observational study investigating the treatment reality of patients with cancer-induced anemia in Germany. It was conducted according to the Declaration of Helsinki, reviewed by an ethics committee, and registered in the ClinicalTrials.gov registry (NCT01795690).

2.2. Patients. Eligible patients were $\geq 18$ years old, with diagnosed cancer, irrespective of tumor type, and about to start antianemic therapy with baseline $\mathrm{Hb}$ levels $<11 \mathrm{~g} / \mathrm{dL}$ (females) or $<12 \mathrm{~g} / \mathrm{dL}$ (males). Antianemic treatment was started no longer than 7 days prior to signing written informed consent. Additional inclusion criteria comprised an Eastern Cooperative Oncology Group performance status of $0-3$ and life expectancy of $>16$ weeks. Patients with myelodysplastic syndrome or an experimental antianemic therapy as part of a clinical trial were excluded. Study sites were encouraged to enroll patients consecutively to ensure unselected recruitment. Patients were treated according to physicians' choice based on patients' individual needs.

2.3. Data Collection. At the time of enrolment, data on patients' sociodemographics, tumor entity, type of antineoplastic treatment, concomitant diseases, previous antianemic treatments, and current laboratory parameters were documented. Comorbidity was assessed using the Charlson Comorbidity Index [16]. During the 12-week observational period, antianemic treatment and laboratory parameters were documented. Data were collected from patients' medical files and transferred to a secure web-based electronic case report form (eCRF) by physicians or trained study nurses. Implemented automatic completeness and plausibility checks, and if necessary direct contact with the study site, were done for quality assurance. To determine QoL, patients completed the Functional Assessment of Cancer Therapy Anemia (FACT-An) questionnaire at enrolment and 6 and 12 weeks later. The initial questionnaire was filled at the study site; the remaining two were mailed to the patients, filled at home, and returned by mail in prepaid envelopes. All patients who returned the baseline questionnaire were included in the analysis of patient-reported outcomes.

2.4. Patient Cohort and Statistical Analysis. Of all patients recruited, those with documented baseline $\mathrm{Hb}$ (measured no longer than 7 days before the start of antianemic treatment) were eligible for the final analysis. Patients who received one of the four standard antianemic treatments (RBCTx, ESA, IV iron, or ESA + IV iron) on day 1 of the observation period (day 1 treatment) were included in the present analysis. Patients were categorized by (1) their type of disease (solid operable tumor/potentially curative, solid inoperable tumor/palliative intention, and hematological tumor) and by (2) their day 1 treatment. The frequency of diagnostic measurements at the start of treatment was calculated. For this purpose, the number of patients for whom specified blood parameters were measured at least once within 4 weeks until 2 weeks after the beginning of antianemic treatment was determined. To analyze the effectiveness of treatments, the proportion of "responders" and $\Delta \mathrm{Hb}$ (final) and $\Delta \mathrm{Hb}$ (max) were determined. $\Delta \mathrm{Hb}$ (final) was defined as the difference between the baseline $\mathrm{Hb}$ and the last $\mathrm{Hb}$ documented within the observation period, but at least 4 weeks after the start of treatment. $\Delta \mathrm{Hb}(\max )$ was defined as the difference between the baseline $\mathrm{Hb}$ and the highest $\mathrm{Hb}$ documented. "Responders" were all patients with final $\mathrm{Hb}$ of $>11 \mathrm{~g} / \mathrm{dL}$ or with $\Delta \mathrm{Hb}$ (final) of $\geq 1.5 \mathrm{~g} / \mathrm{dL}$, with the final $\mathrm{Hb}$ being the last documented $\mathrm{Hb}$ within the observation period, but at least 4 weeks after the start of antianemic treatment. 
The FACT-An total score and the anemia-specific subscale score were determined according to the questionnaire's manual. Missing data within a questionnaire were handled according to the questionnaire's manual [17]. Median scores were calculated for each time point and patient sample. No imputations for missing questionnaires were performed. Improvements of seven points on the FACT-An total scale and four points on the anemia subscale were considered clinically meaningful $[14,15]$. The statistical analysis was performed using STATISTICA (StatSoft, Inc.) version 10.0, R version 2.15.1, and IBM SPSS Statistics version 19.0.

\section{Results and Discussion}

3.1. Patients' Characteristics and Day 1 Treatment. Between March 2012 and September 2013, 216 office-based medical oncologists from 88 study sites recruited 1018 patients. Of these patients, 984 were eligible for analysis. 22 patients were excluded because treatment sample sizes were too small for meaningful analysis. They received nonstandard day 1 treatments (8 oral iron, 5 ESA + RBCTx, 4 oral iron + RBCTx, 2 oral iron + IV iron + RBCTx, 2 IV iron + RBCTx, and 1 oral iron + IV iron), and to this end 962 patients were included in the present study (Figure 1).

Table 1 displays the baseline sociodemographic and clinical characteristics. Overall, $85 \%$ of the patients $(n=813)$ had solid tumors, predominantly breast, colorectal, and nonsmall cell lung cancer (NSCLC), whereas the remaining patients $(15 \%, n=149)$ were affected by hematological malignancies. $75 \%$ of the solid tumors were inoperable (palliative patients, $n=606$ ). Mean baseline $\mathrm{Hb}$ was $8.9 \mathrm{~g} / \mathrm{dL}$.

The majority of patients $(88 \%, n=850)$ received chemotherapy, of which about half were platinum based (Table 1). Figure 2 presents the frequency of the most common day 1 treatments according to type of disease $(n=962)$. Overall, $59 \%(n=571)$ of the patients received RBCTx, 20\% $(n=196)$ underwent ESA therapy, and $15 \%(n=142)$ were treated with IV iron. A combination of ESAs and IV iron was the treatment of choice for the minority of patients $(6 \%$, $n=53$ ) (Table 1). Thus, approximately $40 \%$ of the patients received antianemic therapy with ESA, IV iron, or ESA + IV iron.

Patients with inoperable solid tumors and patients with hematological malignancies were treated more often with RBCTx (60\% and 64\%, resp.) than patients with operable solid tumors (55\%). ESA therapy was used less frequently in patients with inoperable solid tumors than in patients with operable solid tumors and hematological malignancies (18\% versus $25 \%$ ). Of all patients with solid tumors receiving ESAs, patients with breast cancer constitute approximately onethird. $20-30 \%$ of the patients with solid tumors and treated with IV iron had colorectal cancer (Table 1).

Approximately $20 \%$ of the patients had received previous antianemic therapies within 4 weeks before day 1 of the observation period, mostly RBCTx (data on file).

Our data show that in 2012/2013 transfusions accounted for almost $60 \%$ of day 1 antianemic treatments in German routine practice, while ESA (alone or with IV iron) was used in $26 \%$ and IV iron alone in $15 \%$ of patients. In 2004/2005, the

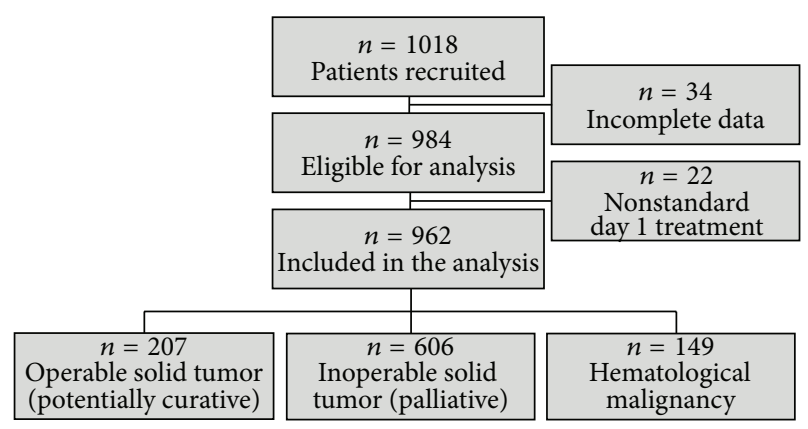

FIGURE 1: Patient recruitment, patient cohort, and type of disease.

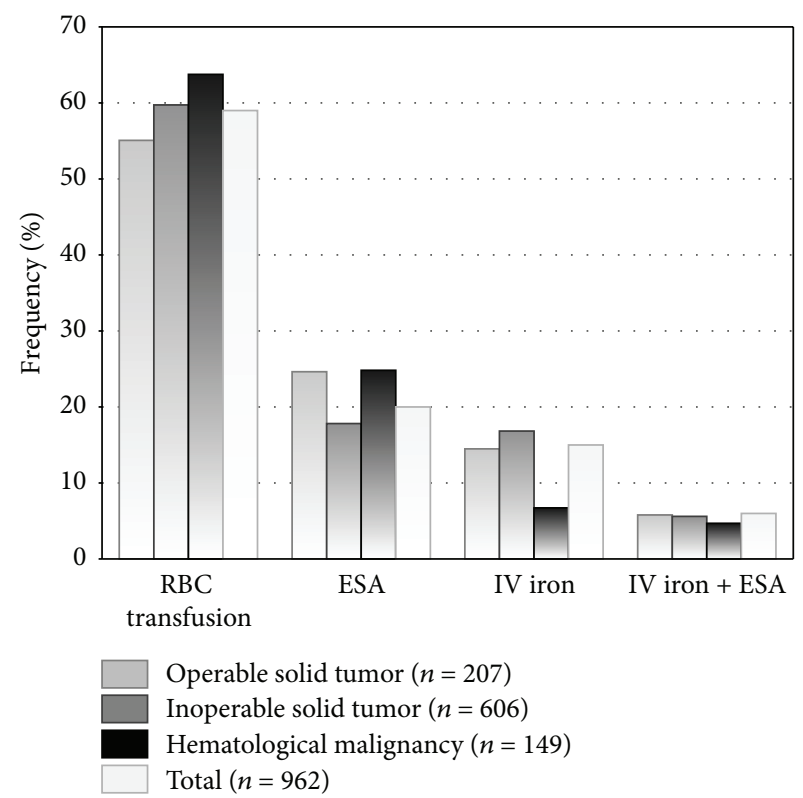

FIGURE 2: Frequency of antianemic day 1 treatments by type of disease.

German CAR study reported that transfusions were planned as "first-line" antianemic therapy for almost 35\% of anemic cancer patients, whereas ESAs were chosen for 39\% and strategies "correcting the underlying disorder" for $26 \%$ of patients [3]. Mean $\mathrm{Hb}$ for requiring treatment was $9.4 \mathrm{~g} / \mathrm{dL}$ in CAR, while mean $\mathrm{Hb}$ at the start of treatment was $8.9 \mathrm{~g} / \mathrm{dL}$ in TAR. Mean $\mathrm{Hb}$ triggering transfusion was $8.7 \mathrm{~g} / \mathrm{dL}$ in CAR, while mean $\mathrm{Hb}$ at the start of transfusion as day 1 treatment was $8.6 \mathrm{~g} / \mathrm{dL}$ in TAR. Mean $\mathrm{Hb}$ when ESAs, IV iron, and ESA + IV iron were chosen was $9.7 \mathrm{~g} / \mathrm{dL}$ in CAR and between 9 and $10 \mathrm{~g} / \mathrm{dL}$ in TAR (data on file).

In 2001/2002, the ECAS reported that approximately $38 \%$ of those patients receiving any antianemic therapy were treated with transfusions (alone or in combination with iron) at any time during the observational period, while approximately $45 \%$ received ESAs (alone or in combination with iron and/or transfusion) [2].

It has to be noted that CAR also included patients with $\mathrm{Hb}$ levels $<12 \mathrm{~g} / \mathrm{dL}$ (females) or $<13 \mathrm{~g} / \mathrm{dL}$ (males), who are less likely to receive transfusions. On the other hand, 33\% of patients in CAR had a lymphoproliferative malignancy 


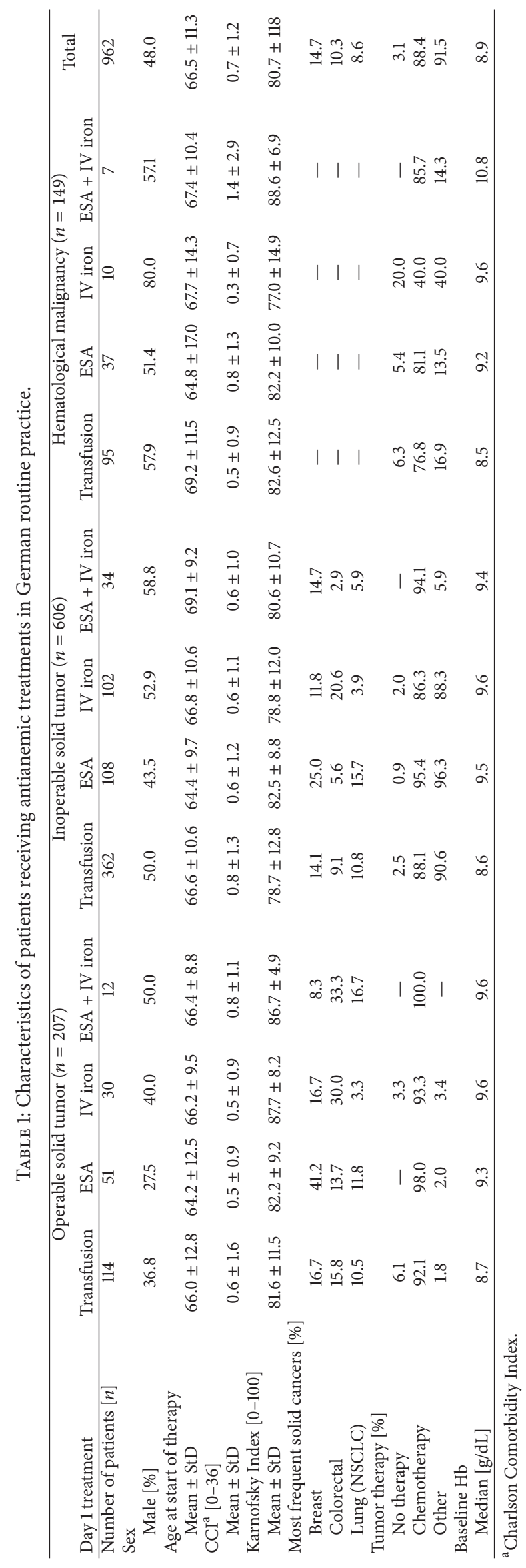


compared to $15 \%$ in TAR, with these patients being more likely to receive transfusions in both data sets. The ECAS recruited all patients, independently of anemia, and also anemic patients that did not require treatment. Mean $\mathrm{Hb}$ at the start of treatment was higher in ECAS $(9.7 \mathrm{~g} / \mathrm{dL})$ than in TAR $(8.9 \mathrm{~g} / \mathrm{dL})$. Patients undergoing chemotherapy and whose $\mathrm{Hb}$ was $<9 \mathrm{~g} / \mathrm{dL}$ at the start of antianemic treatment were treated most frequently with transfusions (53\%). In contrast, $71 \%$ of TAR patients receiving transfusions had $\mathrm{Hb}$ of $<9 \mathrm{~g} / \mathrm{dL}$.

In summary, our data indicate that the use of transfusions as antianemic treatment might have increased in the last decade, while the use of ESA has decreased. Due to the limitations of historic controls, it cannot be excluded that the differences seen in the frequencies of treatments in ECAS, CAR, and TAR are at least partially caused by differences in the design of these studies (inclusion criteria) resulting in different patients recruited (e.g., with lower $\mathrm{Hb}$ in TAR) and thus receiving different treatments (e.g., more transfusions in TAR).

3.2. RBC Transfusions in Patients with $\mathrm{Hb}$ Levels $\geq 8 \mathrm{~g} / \mathrm{dL}$. In general, patients receiving RBCTx as day 1 treatment showed lower baseline $\mathrm{Hb}$ values than patients receiving other antianemic therapies (Table 1).

Overall, $85 \%$ ( $n=813$ ) of all patients had baseline $\mathrm{Hb}$ levels $\geq 8 \mathrm{~g} / \mathrm{dL}$. Of these patients, $55 \%(n=443)$ received RBCTx. This also means that, of all patients receiving transfusions $(n=571)$, almost $80 \%(n=443)$ had baseline $\mathrm{Hb}$ values $\geq 8 \mathrm{~g} / \mathrm{dL}$. Study sites reported the presence of anemic symptoms for $88 \%$ of patients with $\mathrm{Hb}$ levels $\geq 8 \mathrm{~g} / \mathrm{dL}$. In total, $71 \%(n=406)$ of the patients who received transfusions ( $n=571)$ had $\mathrm{Hb}$ values $<9.0 \mathrm{~g} / \mathrm{dL}$ at the start of treatment (data on file).

The high rates of RBCTx, especially in patients with $\mathrm{Hb}$ $\geq 8 \mathrm{~g} / \mathrm{dL}$ in TAR, are concerning, considering the various risks, such as transfusion-transmitted infections, transfusionrelated circulatory overload, iron overload, anaphylactic reactions, and thromboembolism $[8,12,18]$. While RBCTx are the only option when immediate correction of anemic symptoms is required, there is ongoing debate about the $\mathrm{Hb}$ that should trigger transfusions, which is reflected in several changes in guidelines over time [18-20]. According to the EORTC guideline on the use of ESAs, patients should be evaluated for the need of transfusions if their $\mathrm{Hb}$ level is $<9 \mathrm{~g} / \mathrm{dL}$ [21]. Guidelines of the German Medical Association indicate the use of transfusions for patients with symptomatic anemia whose $\mathrm{Hb}$ level is $<8 \mathrm{~g} / \mathrm{dL}$ and/or for patients with symptomatic cardiovascular disease and the additional presence of physiologic transfusion triggers, such as tachycardia or hypotension, along with an $\mathrm{Hb}$ level between 8 and $10 \mathrm{~g} / \mathrm{dL}$ [13]. The high rate of transfusions in patients with $\mathrm{Hb}$ levels $\geq 8 \mathrm{~g} / \mathrm{dL}$ in TAR may only partly be explained by the presence of physiologic transfusion triggers, data on which were not collected within this study. There may be other rationales for applying transfusions more often than other treatments in patients with $\mathrm{Hb}$ levels $\geq 8 \mathrm{~g} / \mathrm{dL}$ and in the study cohort as a whole.

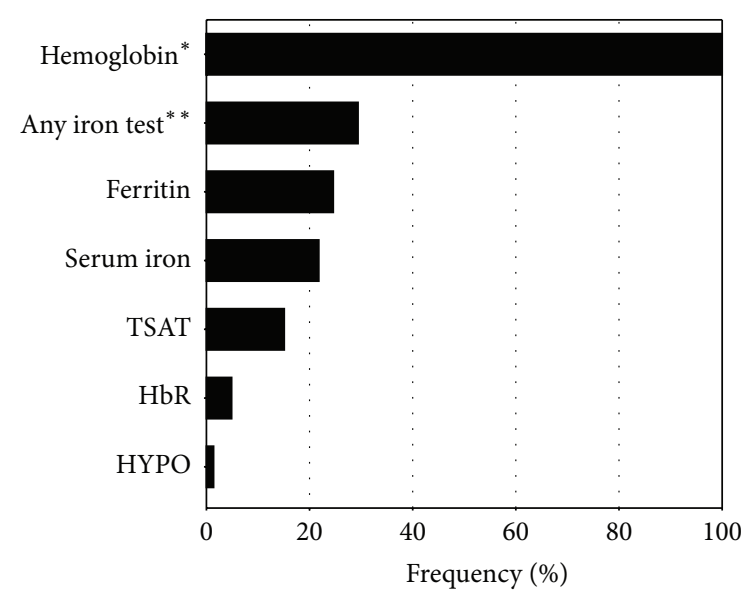

FIGURE 3: Frequency of patients tested for iron parameters at the start of antianemic treatment. ${ }^{*}$ Inclusion criterion; ${ }^{* *}$ Patients tested for any of the listed iron parameters: ferritin, serum iron, TSAT (transferrin saturation), $\mathrm{HbR}$ (hemoglobin content of reticulocytes), or HYPO (hypochromic erythrocytes).

3.3. Testing for Specific Blood Serum Parameters. About a quarter of TAR patients received ESAs as day 1 treatment, either alone or in combination with IV iron. Although a direct comparison is prevented by the reasons mentioned above, findings of the CAR and the ECAS reported a higher use of ESAs in Germany and Europe in 2001-2005 (CAR: planned ESA treatment rate of almost $40 \%$; ECAS: approximately $45 \%$ of the patients were treated with epoetin, either alone or in combination with iron and/or transfusion) [2, 3]. Since then, safety concerns have led to revisions of existing practice guidelines $[10,12]$. A summary of meta-analyses on ESA use in cancer patients from 2011 came to the conclusion that, overall, ESAs reduced the risk for RBCTx and increased the risk for thrombovascular events and mortality, while the effect of ESAs on mortality in patients receiving chemotherapy was unclear [22]. A Cochrane meta-analysis found no evidence for increased mortality in patients with target $\mathrm{Hb}<12 \mathrm{~g} / \mathrm{dL}$, undergoing chemotherapy [23]. Thus, in clinical practice, the benefits and risks of ESAs and transfusions should be carefully considered and decisions should be made based on each patient's situation and preferences.

In this context, it is of great concern that only approximately one-third (30\%) of all patients in TAR were tested for iron parameters at the start of antianemic treatment, most frequently by measuring ferritin, serum iron, or TSAT within 4 weeks before the start of therapy. Testing for $\mathrm{Hb}$ content of reticulocytes and hypochromic erythrocytes occurred even less frequently (Figure 3). Iron parameters were measured more often in patients with colorectal cancer than in patients affected by other malignancies ( $43 \%$ versus $28 \%$, data on file).

Evaluation of nutritional deficiencies other than iron was rarely done; $<1 \%$ of patients were analyzed for deficits in vitamin B12 and folic acid, respectively (data on file).

While it is possible that a proportion of patients had been tested prior to the four weeks before inclusion into TAR, this is unlikely to account for almost $70 \%$ of patients without 
documented diagnostics. Only $20 \%$ of patients had received antianemic treatment in the four weeks prior to inclusion. Data from the CAR reported that $44 \%$ of patients had been tested for ferritin and 33\% for TSAT, although the time frame was not restricted and could have been more than four weeks before treatment [3].

While the NCCN recommends thorough diagnostics for possible treatable causes of anemia, including AID and FID, and specifies the parameters to be tested, no guideline on the diagnostics and treatment of cancer-related anemia has been published in Europe to date. In the TAR study, testing for iron parameters was performed more often in patients with colorectal cancer than in patients affected by other malignancies, accompanied by a higher frequency of IV iron therapy in this patient subgroup. This indicates that physicians currently use diagnostics for specific subsets of patients rather than as a routine requirement prior to any antianemic therapy.

IV iron has been shown to improve the efficacy of ESAs in patients with FID and is thus recommended for this patient subgroup $[10,12]$. In addition, IV or oral iron is the treatment of choice in patients with AID [12]. In the TANDEM study, a diagnostic algorithm to select patients to antianemic treatment was suggested [24] based on the diagnostic plot by C. Thomas and L. Thomas [25] and identified about $25 \%$ of patients with iron deficiency in a cohort primarily designated for ESA treatment.

3.4. Effectiveness. Overall, antianemic treatment was successful in approximately half of all patients ("responders," Table 2). Data on effectiveness are limited by the observational study design. There is considerable heterogeneity between the patients and thus effectiveness of treatments and QoL should not be compared between the different types of therapies. Causal relations cannot be drawn. Patient characteristics and inclusion criteria, such as baseline $\mathrm{Hb}$ levels $<11 \mathrm{~g} / \mathrm{dL}$ (females) or $<12 \mathrm{~g} / \mathrm{dL}$ (males), have to be considered when comparing data with other published studies.

Patients receiving transfusions had median final $\mathrm{Hb}$ between 1.2 and $1.6 \mathrm{~g} / \mathrm{dL}$ above baseline, depending on the type of disease $(\Delta \mathrm{Hb}$ (final), Table 2$)$. The maximum median increase after the start of treatment was between 2.6 and $2.9 \mathrm{~g} / \mathrm{dL}(\Delta \mathrm{Hb}(\max )$, Table 2$)$. Patients receiving ESAs showed median final $\mathrm{Hb}$ between 1.9 and $2.1 \mathrm{~g} / \mathrm{dL}$ above baseline $(\Delta \mathrm{Hb}$ (final), Table 2$)$. The maximum median increase was between 2.6 and $2.7 \mathrm{~g} / \mathrm{dL}$ after the start of treatment $(\Delta \mathrm{Hb}(\max )$, Table 2$)$. Patients with inoperable solid tumors treated solely with IV iron, who in general had higher baseline $\mathrm{Hb}$ values (median $9.6 \mathrm{~g} / \mathrm{dL}$, Table 1 ), showed median final $\mathrm{Hb}$ of $1.1 \mathrm{~g} / \mathrm{dL}$ above baseline. Due to the small number of patients, effectiveness parameters for other subgroups should be interpreted with caution.

The majority of patients receiving no RBCTx as antianemic therapy required no additional transfusions during the observation period (Table 2). According to the criteria defined in this study, all treatments were on average effective within the patient populations investigated. More than half of all patients showed a long-term rise in $\mathrm{Hb}$ levels. The main purpose of antianemic treatment is not only to correct $\mathrm{Hb}$ levels, but also to improve QoL [3].

3.5. Quality of Life. While QoL is being measured more frequently in clinical trials, data on QoL in unselected, reallife patients are still rare. The FACT-An questionnaire is a validated tool to assess QoL in anemic cancer patients and to discriminate patients by their $\mathrm{Hb}$ levels and physical performance status [26]. In total, $78 \%, 70 \%$, and $60 \%$ of the patients returned QoL questionnaires at baseline, after 6 weeks, and after 12 weeks, respectively. Median baseline FACT-An total scores (maximum 188 points) indicating overall QoL were between 104.1 and 115.9 points for all patients, with patients receiving transfusions having the lowest score (Figure 4(a)). Median baseline anemia-specific subscale scores (maximum 80 points) were $<45$ for all patients (transfusion: 41.6, ESA: 44.0, IV iron: 43.5, and ESA + IV iron: 41.0; Figure 4(b)). The median anemia-specific subscale scores showed improvement in all treatment groups. Clinically meaningful changes ( $\geq 4$ points) were observed after 12 weeks for patients receiving ESA ( 44.0 to 48.2 points), IV iron ( 43.5 to 51.3 points), or ESA + IV iron (41.0 to 50.0 points). For patients receiving ESAs, clinically meaningful changes were already observed after 6 weeks ( 44.0 to 48.2 points). Overall QoL, as measured by the FACT-An total scores, also showed a median improvement after 12 weeks for patients receiving IV iron or ESA + IV iron. The difference reached the level of clinical relevance $(\geq 7$ points) for patients receiving ESA + IV iron (106.5 to 117.5 points); however, due to the small number of patients, this result should be interpreted with caution.

On average, a clinically meaningful improvement in the anemia-specific subscale scores was observed for TAR patients undergoing therapies other than RBCTx. However, this has to be interpreted with caution and might not be caused by the treatment applied, since patients receiving transfusions had lower $\mathrm{Hb}$ values at the start of treatment among other differences, which may also affect QoL. Improvement in QoL during antianemic treatment was also recently reported for patients receiving darbepoetin in German routine practice [7].

\section{Conclusion}

The aim of the TAR study was to assess the current treatment of anemia in cancer patients in German routine practice. Our data show that the majority of patients receive RBCTx, while ESAs, IV iron, or a combination of both is applied less frequently. Diagnostic testing for iron or other nutritional deficiencies is not routinely performed before treatment. All antianemic treatments were effective within the patient populations examined. Therefore, our data suggest that diagnostics for possible causes and causal therapies of anemia are underused in German routine practice. The large proportion of patients treated with transfusions, especially with $\mathrm{Hb}$ values $\geq 8 \mathrm{~g} / \mathrm{dL}$, highlights the need for systematic studies on the benefits of diagnostic-led treatment decision-making and for a European guideline on anemia management. It urgently needs to be discussed whether thorough diagnostics and 


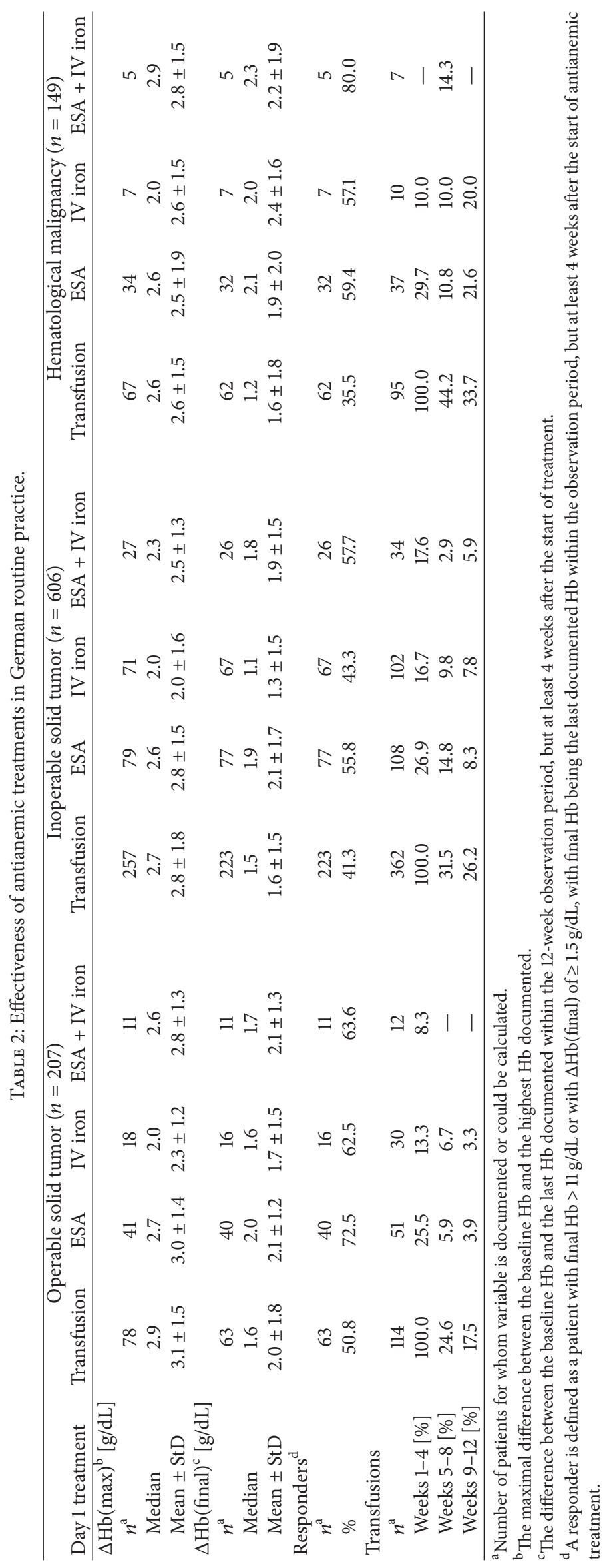




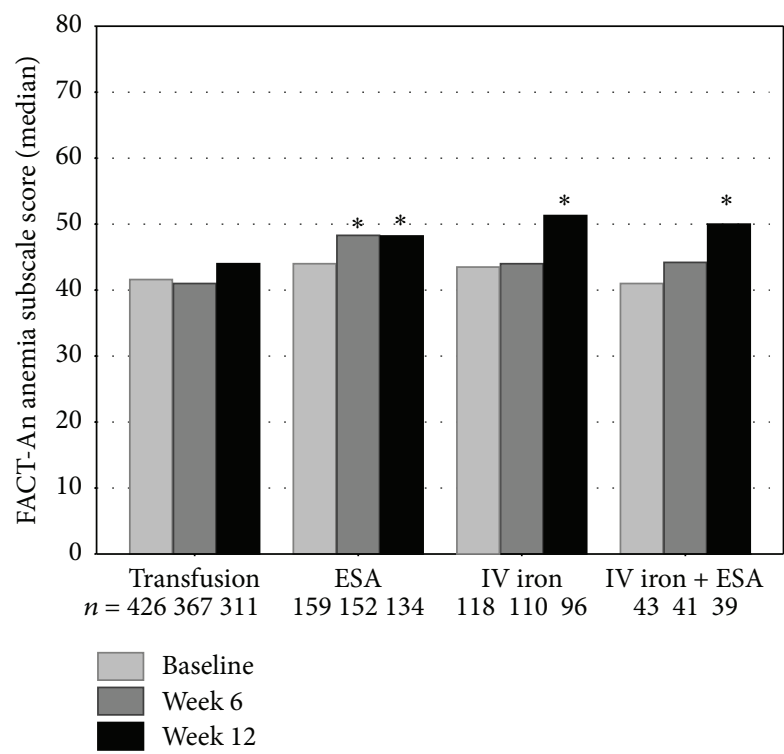

(a)

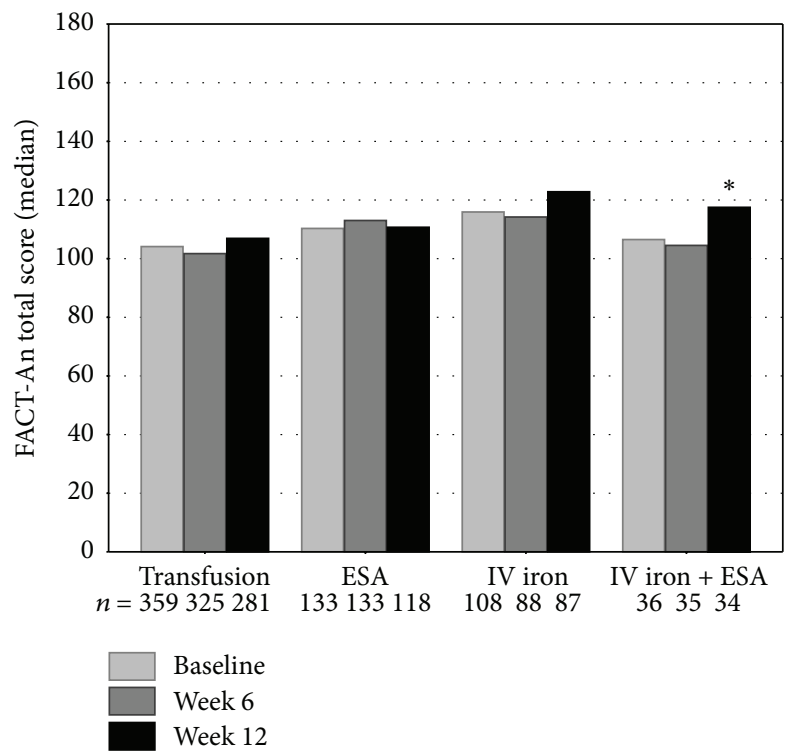

(b)

FIGURE 4: Quality of life (QoL) of patients assessed by the FACT-An questionnaire at baseline and after 6 and 12 weeks of the observation period. (a) Anemia-specific subscale score range [0-80]; ${ }^{*}$ a difference of 4 points is considered clinically relevant [14, 15]; (b) FACT-An total score range [0-188]; ${ }^{*}$ a difference of 7 points is considered clinically relevant [14, 15]; higher scores indicate a better QoL; numbers indicate the number of questionnaires returned.

earlier intervention can decrease the need for transfusions, at least in subsets of patients.

\section{Conflict of Interests}

The authors declare that there is no conflict of interests regarding the publication of this paper.

\section{Acknowledgments}

The authors thank all patients, physicians, and study teams participating in the TAR study. They also thank Vifor Pharma Deutschland $\mathrm{GmbH}$ for the supporting research grant. Moreover, they thank Holger Hartmann and Jörg Spirik (iOMEDICO) and Olof Harlin and Garth Virgin (Vifor Pharma) for support and comments during the design and setup of the project; Martina Jänicke, Anja Kaiser-Osterhues, Lisa Spring, and Susanne Tech (iOMEDICO) for support with the writing of the paper; and Beate Stremmel and Garth Virgin (Vifor Pharma) for critical comments on the paper. The study was conducted in collaboration with the Arbeitsgemeinschaft Supportive Maßnahmen in der Onkologie, Rehabilitation und Sozialmedizin (ASORS).

\section{References}

[1] WHO, "Haemoglobin concentrations for the diagnosis of anaemia and assessment of severity," Vitamin and Mineral Nutrition Information System, Geneva, Switzerland, World Health Organization (WHO/NMH/NHD/MNM/11.1), 2011, http://www.who.int/vmnis/indicators/haemoglobin.pdf.
[2] H. Ludwig, S. Van Belle, P. Barrett-Lee et al., "The European Cancer Anaemia Survey (ECAS): a large, multinational, prospective survey defining the prevalence, incidence, and treatment of anaemia in cancer patients," European Journal of Cancer, vol. 40, no. 15, pp. 2293-2306, 2004.

[3] T. Steinmetz, U. Totzke, M. Schweigert et al., "A prospective observational study of anaemia management in cancer patients-results from the German Cancer Anaemia Registry," European Journal of Cancer Care, vol. 20, no. 4, pp. 493-502, 2011.

[4] G. Birgegård, P. Gascón, and H. Ludwig, "Evaluation of anaemia in patients with multiple myeloma and lymphoma: findings of the European CANCER ANAEMIA SURVEY," European Journal of Haematology, vol. 77, no. 5, pp. 378-386, 2006.

[5] P. J. Barrett-Lee, H. Ludwig, G. Birgegård et al., "Independent risk factors for anemia in cancer patients receiving chemotherapy: results from the European Cancer Anaemia Survey," Oncology, vol. 70, no. 1, pp. 34-48, 2006.

[6] J. Bohlius, T. Tonia, E. Nüesch et al., "Effects of erythropoiesisstimulating agents on fatigue- and anaemia-related symptoms in cancer patients: systematic review and meta-analyses of published and unpublished data," British Journal of Cancer, vol. 111, no. 1, pp. 33-45, 2014.

[7] T. Steinmetz, M. Kindler, O. Lange, U. Vehling-Kaiser, A. Kuhn, and E. Hellebrand, "A prospective cohort study on the impact of darbepoetin alfa on quality of life in daily practice following anemia treatment guideline revisions," Current Medical Research and Opinion, vol. 30, no. 9, pp. 1813-1820, 2014.

[8] J. L. Spivak, P. Gascón, and H. Ludwig, "Anemia management in oncology and hematology," Oncologist, vol. 14, supplement 1, pp. 43-56, 2009.

[9] D. Cella, M. J. Zagari, C. Vandoros, D. D. Gagnon, H.-J. Hurtz, and J. W. R. Nortier, "Epoetin alfa treatment results in clinically 
significant improvements in quality of life in anemic cancer patients when referenced to the general population," Journal of Clinical Oncology, vol. 21, no. 2, pp. 366-373, 2003.

[10] M. S. Aapro and H. Link, "September 2007 update on EORTC guidelines and anemia management with erythropoiesisstimulating agents," Oncologist, vol. 13, supplement 3, pp. 33-36, 2008.

[11] J. D. Rizzo, M. Brouwers, P. Hurley et al., "American Society of Clinical Oncology/American Society of Hematology clinical practice guideline update on the use of epoetin and darbepoetin in adult patients with cancer," Journal of Clinical Oncology, vol. 28, no. 33, pp. 4996-5010, 2010.

[12] NCCN, NCCN Clinical Practice Guidelines in Oncology: Cancerand Chemotherapy-Induced Anemia, Version 2.2015, National Comprehensive Cancer Network, 2015, http://www.nccn.org/ professionals/physician_gls/pdf/anemia.pdf.

[13] Bundesärztekammer BÄK (German Medical Association), "Cross-sectional guidelines for therapy with blood components and plasma derivatives-4th revised edition," Transfusion Medicine and Hemotherapy, vol. 36, pp. 345-492, 2009.

[14] K. Webster, D. Cella, and K. Yost, “The Functional Assessment of Chronic Illness Therapy (FACIT) measurement system: properties, applications, and interpretation," Health and Quality of Life Outcomes, vol. 1, article 79, 2003.

[15] J. Ringash, B. O'Sullivan, A. Bezjak, and D. A. Redelmeier, "Interpreting clinically significant changes in patient-reported outcomes," Cancer, vol. 110, no. 1, pp. 196-202, 2007.

[16] M. E. Charlson, P. Pompei, K. L. Ales, and C. R. MacKenzie, "A new method of classifying prognostic comorbidity in longitudinal studies: development and validation," Journal of Chronic Diseases, vol. 40, no. 5, pp. 373-383, 1987.

[17] FACIT.org, "FACT-An Scoring Guidelines," Version 4, May 2003.

[18] A. Calabrich and A. Katz, "Management of anemia in cancer patients," Future Oncology, vol. 7, no. 4, pp. 507-517, 2011.

[19] M. L. Thomas, "Anemia and quality of life in cancer patients: impact of transfusion and erythropoietin," Medical Oncology, vol. 15, supplement 1, pp. S13-S18, 1998.

[20] P. J. Barrett-Lee, N. P. Bailey, M. E. R. O’Brien, and E. Wager, "Large-scale UK audit of blood transfusion requirements and anaemia in patients receiving cytotoxic chemotherapy," British Journal of Cancer, vol. 82, no. 1, pp. 93-97, 2000.

[21] C. Bokemeyer, M. S. Aapro, A. Courdi et al., "EORTC guidelines for the use of erythropoietic proteins in anaemic patients with cancer: 2006 update," European Journal of Cancer, vol. 43, no. 2, pp. 258-270, 2007.

[22] T. Tonia and J. Bohlius, "Ten years of meta-analyses on erythropoiesis-stimulating agents in cancer patients," in Hematopoietic Growth Factors in Oncology, vol. 157 of Cancer Treatment and Research, pp. 217-238, Springer, Berlin, Germany, 2011.

[23] T. Tonia, A. Mettler, N. Robert et al., "Erythropoietin or darbepoetin for patients with cancer," Cochrane Database of Systematic Reviews, vol. 12, Article ID CD003407, 2012.

[24] H. T. Steinmetz, A. Tsamaloukas, S. Schmitz et al., "A new concept for the differential diagnosis and therapy of anaemia in cancer patients," Supportive Care in Cancer, vol. 19, no. 2, pp. 261-269, 2010.

[25] C. Thomas and L. Thomas, "Biochemical markers and hematologic indices in the diagnosis of functional iron deficiency," Clinical Chemistry, vol. 48, no. 7, pp. 1066-1076, 2002.
[26] S. B. Yellen, D. F. Cella, K. Webster, C. Blendowski, and E. Kaplan, "Measuring fatigue and other anemia-related symptoms with the Functional Assessment of Cancer Therapy (FACT) measurement system," Journal of Pain and Symptom Management, vol. 13, no. 2, pp. 63-74, 1997. 


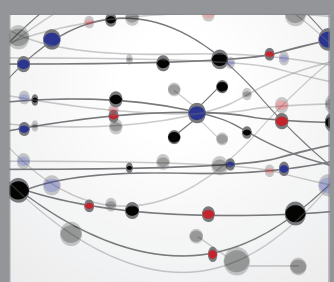

The Scientific World Journal
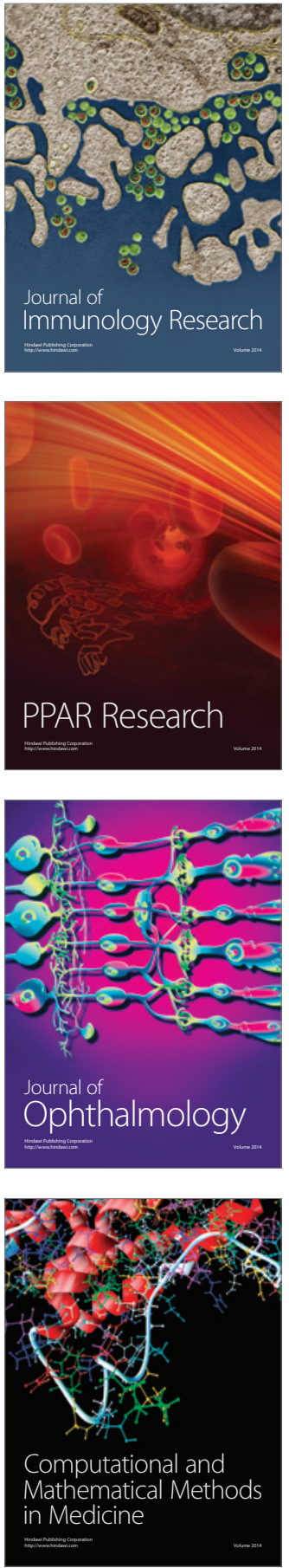

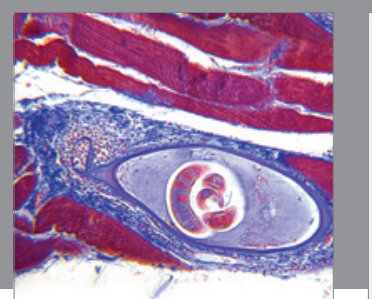

Gastroenterology Research and Practice

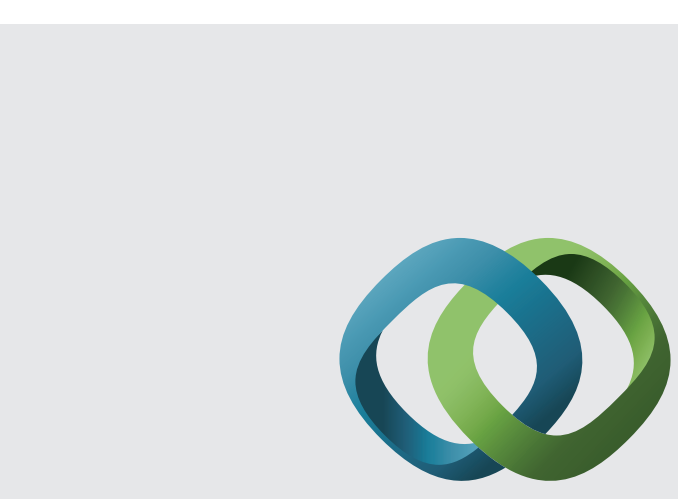

\section{Hindawi}

Submit your manuscripts at

http://www.hindawi.com
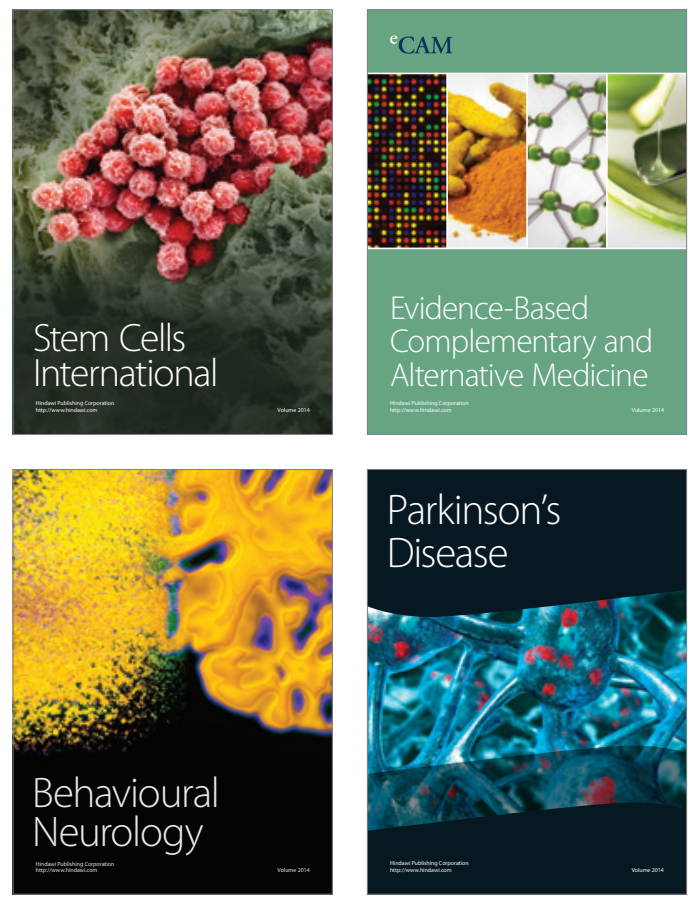
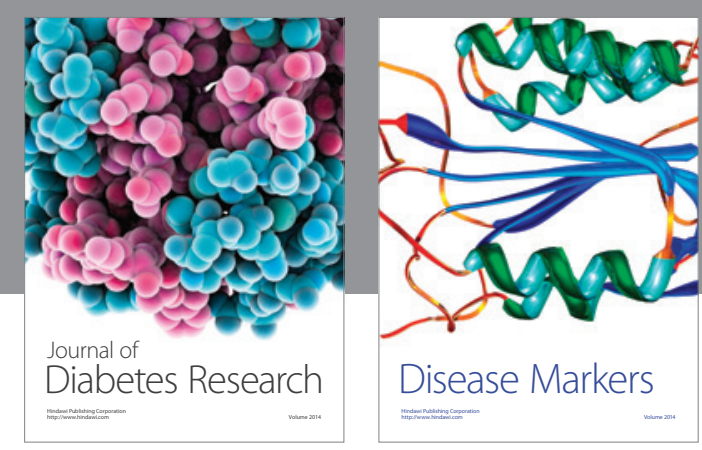

Disease Markers
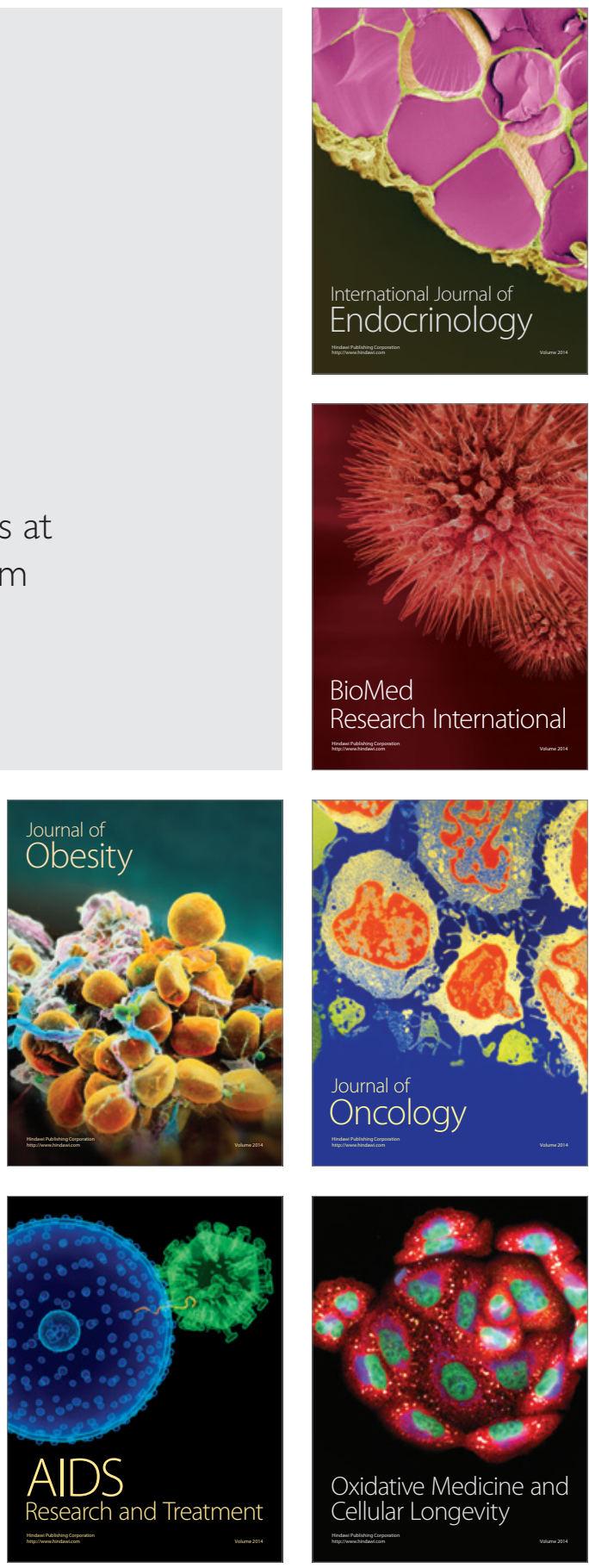